\title{
Observations on the Chromatinic Bodies of Two Species of the Actinoplanaceae
}

\author{
By C. E. BLAND AND J. N. COUCH \\ Department of Botany, University of North Carolina, \\ Chapel Hill, North Carolina 27514, U.S.A.
}

(Accepted for publication I8 March 1968 )

\begin{abstract}
SUMMARY
The structure and distribution of the chromatinic bodies of two species of the genus Actinoplanes were studied with light microscopy. Results obtained from observation of material stained by the technique of DeLamater (I95I), Feulgen (Rafalko, 1946), or Piéchaud (1954), were supplemented by observation of living material. A technique for observing living and stained preparations with phase-contrast or dark-field microscopy gave results far superior to those obtained with bright-field microscopy. Both strains studied had a nearly identical structure and distribution of chromatinic bodies. Colonies consisted of two hyphal types; substrate hyphae and palisade hyphae (Couch, 1950). The chromatinic bodies of the substrate hyphae appeared variously as short rods or as small bands of material that extended across the width of the hyphae. The palisade hyphae contained bodies ranging from dumb-bell shaped structures to multiple entities composed of 3-4 interconnected subunits. The distal portions contained dense rods of chromatinic material. The chromatinic rods of the palisade hyphae progressed into the sporangium along with the ingrowth of the sporogenic hyphae into the sporangial envelope. Within the sporangium these rods appeared to contract and undergo repeated divisions into smaller subunits; a single subunit was incorporated into each spore. A striking similarity in structure and distribution of the chromatinic bodies was noted between members of the Actinoplanaceae and members of the Streptomycetaceae.
\end{abstract}

\section{INTRODUCTION}

The structure and distribution of the chromatinic bodies in the genus Streptomyces (sensu Waksman \& Henrici, 1943) were studied by Badian (1936), Klieneberger-Nobel (1947), McGregor (I954), Saito \& Ikeda (I958), Hopwood \& Glauert (1960) and Shamina (1964). Couch (I954), who used the nuclear staining techniques of Robinow (194I) and DeLamater (I95I), reported chromatinic bodies in Actinoplanes (strain no. 20) to be similar to those found by Klieneberger-Nobel (1947) is Streptomyces, however, no pictures were presented. The purpose of the present paper is to give an illustrated account of the structure and distribution of the chromatinic bodies in two species of the Actinoplanaceae and to compare these findings with those reported by various authors for Streptomyces.

\section{METHODS}

Two species of the genus Actinoplanes were selected for study. One of these, strain 20, was selected by Couch (I954) because it formed abundant vertical palisade hyphae and produced abundant sporangia on a variety of culture media. This strain, no. 20, 
forms its spores in vertical rows within the sporangia (Pl. 2, fig. I I Pl. 4, fig. 27). The other strain, I668, forms its spores in branched coils within the sporangia. Strain I 668 was isolated from soil collected in Hyderabad, South India, whereas strain 20 was isolated from soil collected near Tuscaloosa, Alabama, U.S.A. Both strains will be described in a later publication.

Stock cultures of the two strains were maintained in pure culture on Czapek agar (Fisher Scientific, Fairlawn, New Jersey, U.S.A.). For enhancement of sporulation, cultures were at times grown on the surface of a grass-water medium prepared by autoclaving $30 \mathrm{~g}$. Paspalum grass in $\mathrm{I} 1$. water.

Living material was prepared for study by squashing a small portion of a sporulating colony from either agar or liquid culture in water under a coverslip. To increase the contrast of the chromatinic bodies, living material was mounted in 5-30\% (w/v) concentrations of gelatin and studied with phase-contrast microscopy (Mason \& Powelson, 1956). Similar observations were made in Zeiss W-15 non-hardening mounting medium for phase-contrast microscopy.

For staining, a small block $(\mathrm{I} \times \mathrm{I} \times \mathrm{I}$ o $\mathrm{mm}$. long) from a sporulating culture was crushed under a coverslip, the coverslip removed and the crushed material was fixed for 3-5 min. in the vapour of $2 \%(\mathrm{w} / \mathrm{v})$ osmic acid. This material was fixed to the slide with Haupt's adhesive and allowed to dry. After drying, the slides were placed in distilled water for $5 \mathrm{~min}$. The chromatinic bodies were stained by the technique of DeLamater (I95I), Feulgen (Rafalko, 1946), or Piéchaud (1954). For the Feulgen and DeLamater methods, the preparations were hydrolysed with $\mathrm{N}-\mathrm{HCl}$ for $10 \mathrm{~min}$., then rinsed in distilled water. Feulgen preparation: the preparations were rinsed in sulphurous acid for $2 \mathrm{~min}$., stained in Grübler's leuco-basic fuchsin for $2 \mathrm{hr}$, rinsed in sulphurous acid for $2 \mathrm{~min}$., differentiated in tap water for $15 \mathrm{~min}$., dehydrated in a graded ethanol/water series, and mounted in Zeiss hardening mounting medium for phase-contrast microscopy. DeLamater preparation: the preparations were stained for $2.5 \mathrm{hr}$ in a solution prepared by adding I drop thionyl chloride to Io $\mathrm{ml}$. $0.5 \%(\mathrm{w} / \mathrm{v})$ thionin, rinsed in distilled water, dehydrated in a graded ethanol/water series, and mounted in Zeiss hardening mounting medium for phase-contrast microscopy. The Piéchaud stain was prepared by adding 2 drops $0.5 \%(\mathrm{w} / \mathrm{v})$ eosin-Y to 20 drops of G. T. Gurr's Giemsa stain just before use. The fixed material was stained for 30-60 min. After staining the preparations were mounted in Zeiss W-I5 nonhardening mounting medium for phase-contrast microscopy.

Observations were made with a Zeiss Photomicroscope equipped with a $12 \mathrm{~V}$, 60 W. lamp. Photographs were taken on Kodak Panatomic-X film at magnifications ranging from $\times 160$ to $\times 500$. A Zeiss no. 467806 interference wide-band green filter was used for the phase-contrast and bright-field photographs.

\section{RESULTS}

The mounting of living material in gelatin greatly increased the contrast of the chromatinic bodies; however, better results were obtained by mounting the living material in Zeiss W-I5 non-hardening medium for phase-contrast microscopy. This mounting medium so greatly increased the contrast of the chromatinic bodies when viewed with phase-contrast microscopy that the bodies appeared as if they were stained. When compared with living hyphae mounted in water, no noticeable distortions which 
could be attributed to the Zeiss medium were observed in the structure of the cells or cellular contents of the hyphae.

After staining by the Piéchaud, DeLamater, or Feulgen techniques, the nuclear material was at first very distinct and deeply stained, but all the stains faded very rapidly and within $24 \mathrm{hr}$ the chromatinic bodies were barely visible with bright-field microscopy. These same faded preparations, when observed with either phase-contrast or dark-field microscopy, exhibited chromatinic bodies that were more distinct than when originally observed with bright-field mirocopy. These methods of observations were somewhat better for the Feulgen preparations than for the DeLamater or Piéchaud preparations.

The structure and distribution of the chromatinic bodies of both strains studied were so nearly identical that a detailed description of the findings for one strain makes unnecessary separate descriptions of the two strains. Strain 20 usually gave slightly better staining results and was most often used to make illustrations.

As in Streptomyces sp. (Klieneberger-Nobel, I947), the colonies of the Actinoplanaceae are composed of two distinct hyphal types (Couch, 1950): substrate and palisade hyphae (P1. I, fig. I). The substrate and palisade hyphae seemed to be homologous respectively with the primary and secondary hyphae of Streptomyces sp., as described by Klieneberger-Nobel (I947). The substrate hyphae were branched and thinner than the palisade hyphae (Pl. I, fig. I-3). The palisade hyphae arose vertically as simple branches from the substrate hyphae, as reported for Streptomyces sp. by Hopwood (1960) and Shamina (1964), and grew to the agar surface where they were characteristically terminated by sporangia. The formation of an 'initial cell' as reported by Klieneberger-Nobel (1947) for Streptomyces sp. was not observed.

The chromatinic bodies of the substrate hyphae appeared essentially the same whether mounted in the Zeiss non-hardening mounting medium while living or stained by any of the three methods used (P1. I, fig. 2, 3). The chromatinic masses existed in a wide variety of sizes and shapes. Variation ranged from short rods to small bands of material that extended across the width of the hyphae (P1. I, fig. 2). Septa divided the hyphae into cells which included from I to 4 of the chromatinic bodies. Although there was considerable diversity in size and shape of the chromatinic bodies of the substrate hyphae, they were usually small dense bodies which were markedly similar to the chromatinic bodies described for the substrate hyphae of Streptomyces coelicolor (Hopwood \& Glauert, 1960).

As with the substrate hyphae, all the methods of study yielded a similar structure and distribution of the chromatinic bodies in the palisade hyphae. The palisade hyphae were characterized by having two distinct types of chromatinic bodies (Pl. 2, fig. 4-9; P1. 4, fig. 20-27). The lower half to two-thirds of a palisade hypha contained chromatinic bodies that were very complex and considerably larger than those in the substrate hyphae. These bodies varied in shape from dumb-bell shaped structures to multiple entities composed of 3 to 4 interconnected subunits (P1. 2, fig. 4-6; P1. 4, fig. 20-27). Individual cells of the hyphae contained $\mathrm{I}-6$ such bodies.

The upper portions of the palisade hyphae contained dense rods of chromatinic material that appeared of such a size as to be the equivalent of several of the chromatinic bodies seen in the lower portions of the palisade hyphae (P1. 2, figs. 8, 9; Pl. 4, fig. 20-27). Similar rod-shaped structures were reported by Hopwood \& Glauert (1960) for Streptomyces coelicolor. Chromatinic bodies that resembled elongate rods 
were observed in the upper portion of young palisade hyphae prior to (Pl. 2, fig. 8; Pl. 4, fig. 20), during (Pl. 2, fig. 9; Pl. 4, fig. 2I-26), and after sporangial formation (Pl. 4, fig. 27). Whether the rods are a result of fusion and/or elongation of the chromatinic bodies found in the lower portion of the palisade hyphae was not determined.

The squashing of young and mature sporangia (Pl. 2, fig. IO, I I) under coverslips frequently resulted in the disruption of the sporangium (Pl. 2, fig. 12). With the rupture of the sporangial wall, the sporogenic hyphae contained therein were spread out (Pl. 2, fig. I2) in a manner that greatly facilitated the study of the chromatinic bodies present in the sporangia.

With the formation of the sporangia, the chromatinic rods of the palisade hyphae were found to progress into the sporangium along with the growth of sporogenic hyphae into the sporangial envelope (Pl. 2, fig. 8, 9; Pl. 3, fig. I3, I4; Pl. 4, fig. 2I-24). In strain 20 this process continued until the sporangium was filled with parallel rows of sporogenic hyphae, each of which contained a dense rod of chromatinic material (Pl. 3, fig. 13; Pl. 4, fig. 23, 24). In strain 1668 the sporangium was filled with a coil of branched sporogenic hyphae with each hypha containing a dense rod of chromatinic material. Within the sporogenic hyphae of both strains, the rods of chromatinic material appeared to contract and undergo repeated divisions into smaller subunits (Pl. 3, fig. 15, I6; Pl. 4, fig. 25, 26). These subunits were observed as paired dense bodies within the threads of sporogenic hyphae (Pl. 3, fig. I7, I8; P1. 4, fig. 26). Crosswalls were formed at wide intervals between these bodies (Pl. 3, fig. 17). Further septation of the sporogenic hyphae resulted in only one chromatinic body being included between any two septa. Constrictions occurring simultaneously at the septa produced chains of spores within the sporangium (P1. 2, fig. II; Pl. 4, fig. 27).

Each liberated, mature spore contained a single, round chromatinic body which, as is generally the case with bacterial spores that have been hydrolysed, appeared to have collapsed and formed a crescent along one side of the spore wall (P1. 3, fig. I9).

\section{DISCUSSION}

The technique for observing stained preparations with phase-contrast or dark-field microscopy was recently discovered while examining Feulgen preparations made by Couch in 1953. These preparations were completely faded when observed with brightfield microscopy. However, when viewed with phase-contrast or dark-field microscopy they exhibited chromatinic bodies that were considerably more distinct than when observed as unfaded preparations in 1953 with bright-field microscopy. It appeared that the different refractive index of the stained but faded chromatinic bodies, although not detectable with bright-field microscopy, was greatly enhanced by either phasecontrast or dark-field miroscopy. Even though this technique for observing stained, but faded, preparations was slightly better for the Feulgen stain than for the other stains used, this technique might be found useful by other investigators who at times may need to study stained preparations that have faded.

The structure and distribution of the chromatinic bodies in the living and stained preparations of the Actinoplanes strains studied were almost identical. The Zeiss W-I 5 non-hardening mounting medium proved to be a useful mountant for viewing material with phase-contrast or dark-field microscopy, and did not create any distortions in 
the cells of the hyphae. Hopwood \& Glauert (I960) reported that chromatinic bodies of Streptomyces coelicolor when stained with the Piéchaud technique, which obviates the necessity of preliminary acid hydrolysis, were sharper and hence had more definite outlines than when stained with either the DeLamater or Feulgen techniques. However, the Piéchaud method as used in the present work yielded results that were not noticeably different from those obtained with either the DeLamater or Feulgen technique.

The two strains considered here, as well as other members of the Actinoplanaceae that have undergone preliminary observation, all exhibited striking similarity in structure and distribution of the chromatinic bodies to that reported for members of the Streptomycetaceae (Badian, 1936; Klieneberger-Nobel, 1947; Saito \& Ikeda, 1958; Hopwood \& Glauert, 1960; Shamina, 1964). This similarity between the chromatinic bodies of members of the Actinoplanaceae and those of the Streptomycetaceae corresponds with results reported by Couch (1954). Chromatinic bodies similar to those reported to occur at each major stage in the life-cycle of streptomycetes were found to occur at apparently homologous stages in the life-cycle of strains of the Actinoplanaceae. Strains included in both of these families exhibit two distinct hyphal types: the substrate hyphae, and the palisade or secondary hyphae. The substrate hyphae of the Actinoplanaceae are similar to those of the Streptomycetaceae in frequency of branching, in diameter of hyphae, and in variability of chromatinic bodies. The palisade hyphae of the Actinoplanaceae are similar to the secondary hyphae of streptomycetes in that in both they arise as simple branches from the substrate hyphae, lack numerous branches, are larger than the substrate hyphae, and contain large and complex chromatinic bodies. The sporogenic hyphae of the two families go through very similar stages in the process of sporogenesis. Indeed, if one were to remove the sporangial wall from the sporogenic hyphae of some members of the Actinoplanaceae, it would be difficult to distinguish these organisms from members of the Streptomycetaceae. This similarity in structure and distribution of the chromatinic bodies in these two groups indicates a close relationship between them.

The results presented in this paper seem to be corroborated in the electron micrographs of thin sections of representatives of the Actinoplanaceae by Lechevalier, Lechevalier \& Holbert (1966). Although these authors did not mention chromatinic material, electron-transparent regions within the sporogenic hyphae shown in their micrographs probably represent the chromatinic material. These electron transparent regions appeared to undergo processes similar to those described in this paper.

This work was supported by grants from the National Institutes of Health, 0783I U.S.A., and The Eli Lilly Company.

\section{REFERENCES}

Badian, J. (1936). Über die zytologishe Struktur und den Entwicklungszyklus der Actinomyceten. Acta Soc. bot. polon. 13, 105.

Couch, J. N. (1950). Actinoplanes, a new genus of the Actinomycetales. J. Elisha Mitchell Sci. Soc. 66,87 .

Couch, J. N. (1954). The genus Actinoplanes and its relatives. Trans. N.Y. Acad. Sci. 16, 315.

DeLAMATER, E. D. (1951). A staining and dehydrating procedure for the handling of micro-organisms. Stain Technol. 26, 199.

Hopwoon, D. A. (1960). Phase-contrast observations on Streptomyces coelicolor. J. gen. Microbiol. 22, 295. 
Hopwood, D. A. \& GlauerT, A. M. (1960). Observations on the chromatinic bodies of Streptomyces coelicolor. J. biochem. biophys. Cytol. 8, 257.

KLIENeberger-Nobel, E. (1947). The life cycle of sporing Actinomycetes as revealed by a study of their structure and septation. J. gen. Microbiol. I, 22.

Lechevalier H., Lechevalier, M.P. \& Holbert, P. E. (I966). Electron microscopic observation of the sporangial structure of strains of Actinoplanaceae. J. Bact. 92, 1228.

Mason, D. J. \& Powelson, D. M. (I956). Nuclear division as observed in live bacteria by a new technique. J. Bact. 7r, 474.

McGregor, J. F. (I954). Nuclear division and the life cycle in a Streptomyces sp. J. gen. Microbiol. II, 52.

PiÉCHAud, M. (1954). La coloration sans hydrolyse du noyau des bactéries. Annnls Inst. Pasteur, Paris 86, 787 .

Rafalko, J. S. (1946). A modified Feulgen technic for small and diffuse chromatin elements. Stain Technol. 20-2r, 9r.

Robinow, C. F. (I94I). A study of the nuclear apparatus of bacteria. Proc. R. Soc. B r3o, 299.

Saito, H. \& Ikeda, Y. (1958). The life cycle of Streptomyces griseoflavus. Cytologia 23, 496.

Shamina, Z. B. (1964). Observations on nuclear elements in Actinomyces in the course of spore formation. Mikrobiologiya 33, $83 \mathrm{r}$.

Waksman, S. A. \& Henrici, A. T. (I943). The nomenclature and classification of the Actinomycetes. J. Bact. 46, 337.

\section{EXPLANATION OF PLATES}

Plate I

Fig. I. Strain I668 from Czapek agar culture. Feulgen stain, phase-contrast. Palisade hyphae $(P)$, substrate hyphae $(S)$, origins of plaisade hyphae (arrows), sporangia $(S P) . \times 2000$.

Fig. 2, 3. Substrate hyphae of strain 20. Fig. 2, Feulgen stain, phase-contrast, $\times 1,000$. Fig. 3, Zeiss W-I 5 non-hardening mounting medium, $\times$ I 330 . Chromatinic bodies $(C)$.

\section{Plate 2}

Fig. 4-6. Chromatinic bodies in lower portions of palisade hyphae. Feulgen stain, phase-contrast. Fig. 4, $\times 1200$. Fig. 5, 6, $\times 4000$.

Fig. 7. Young palisade hypha. Feulgen stain, phase-contrast, Proximal chromatinic bodies $(X)$, distal chromatinic bodies $(Y)$, origin of palisade hypha (arrow). $\times 1500$.

Fig. 8, 9. Distal portions of young palisade hyphae. Feulgen stain, phase-contrast. Distal thread of chromatin $(Y)$, young sporangia $(S), \times 2200$.

Fig. IO-I2. Sporangia of strain 20. Fig. IO, Young sporangium with sporogenic hyphae, $\times 1400$.

Fig. I I, Mature sporangia filled with spores, $\times$ I000. Fig. I2, Open sporangium exposing sporogenic hyphae, $\times$ I000. Phase-contrast. Sporangial wall $(W)$.

\section{Plate 3}

Fig. 13-17. Sporogenic hyphae from sporangia of strain 20. Feulgen stain, phase-contrast. Chromatinic material $(C)$, septa $(S)$.

Fig. 13, Dense rods of chromatin inside sporogenic hyphae. $\times 4000$. Fig. 14, Sporogenic hyphae spread out to show distribution of chromatinic material, $\times 2040$. Fig. 15, 16, Division of chromatinic bodies into smaller subunits, $\times 3600$. Fig. 17, Paired chromatinic bodies inside sporogenic hyphae. $\times 2600$.

Fig. 18. Sporogenic hyphae broken from a sporangium at the tip of a palisade hyphae. Feulgen stain, dark-field. Chromatinic bodies $(C)$. $\times 1680$.

Fig. 19. Spores of strain 20. Feulgen stain, phase-contrast. Chromatinic bodies $(C) . \times 3250$.

\section{Plate 4}

Fig. 20-27. Semi-diagrammatic drawings of palisade hyphae of strain 20 to show chromatinic bodies at various stages during sporangial formation. Chromatinic material is stippled. Hyphae are nearly straight but shown curved to conserve space. $\times$ I 400 . 

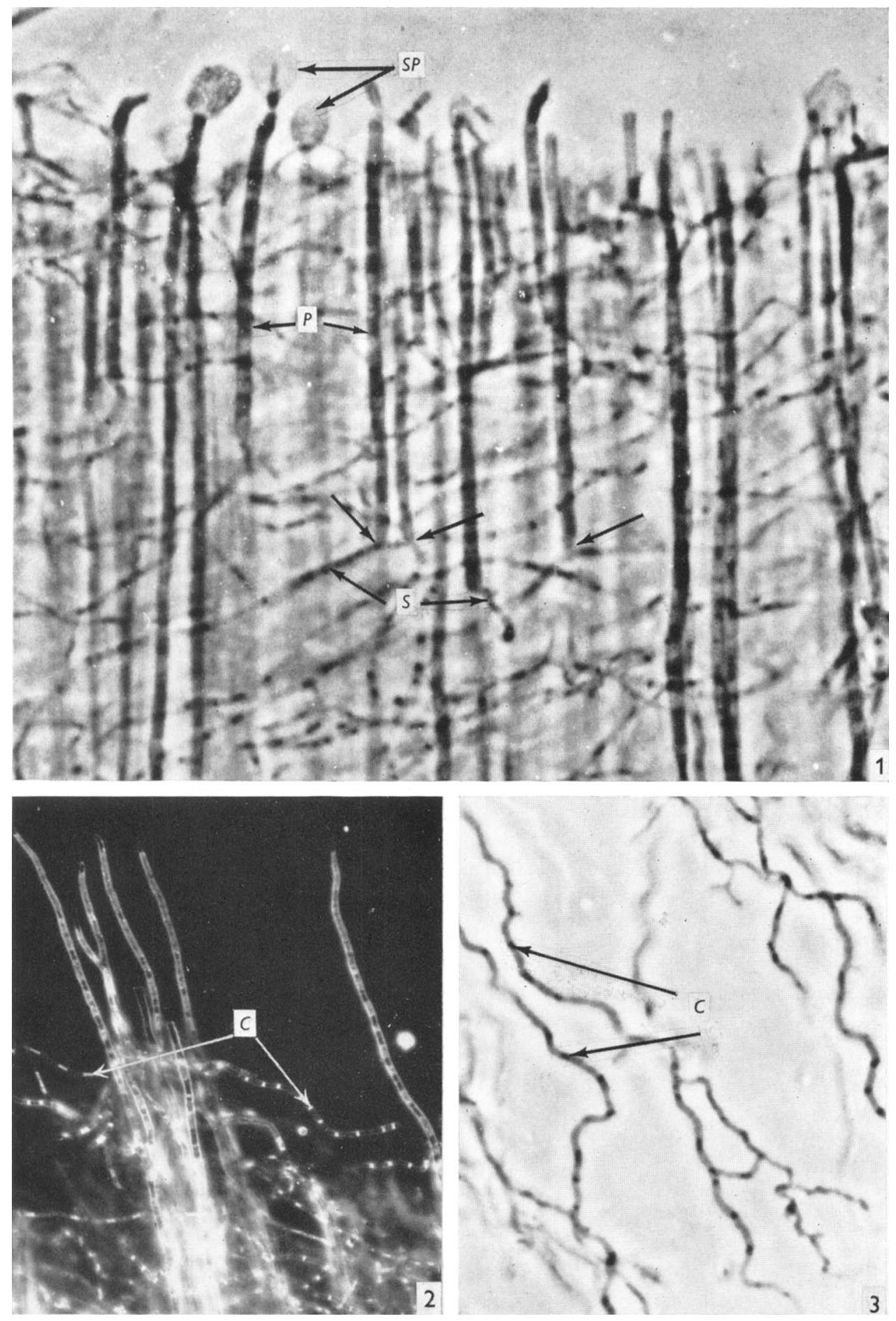

C. E. BLAND AND J. N. COUCH

(Facing p. 100) 
Journal of General Microbiology, Vol. 53, No. I

Plate 2
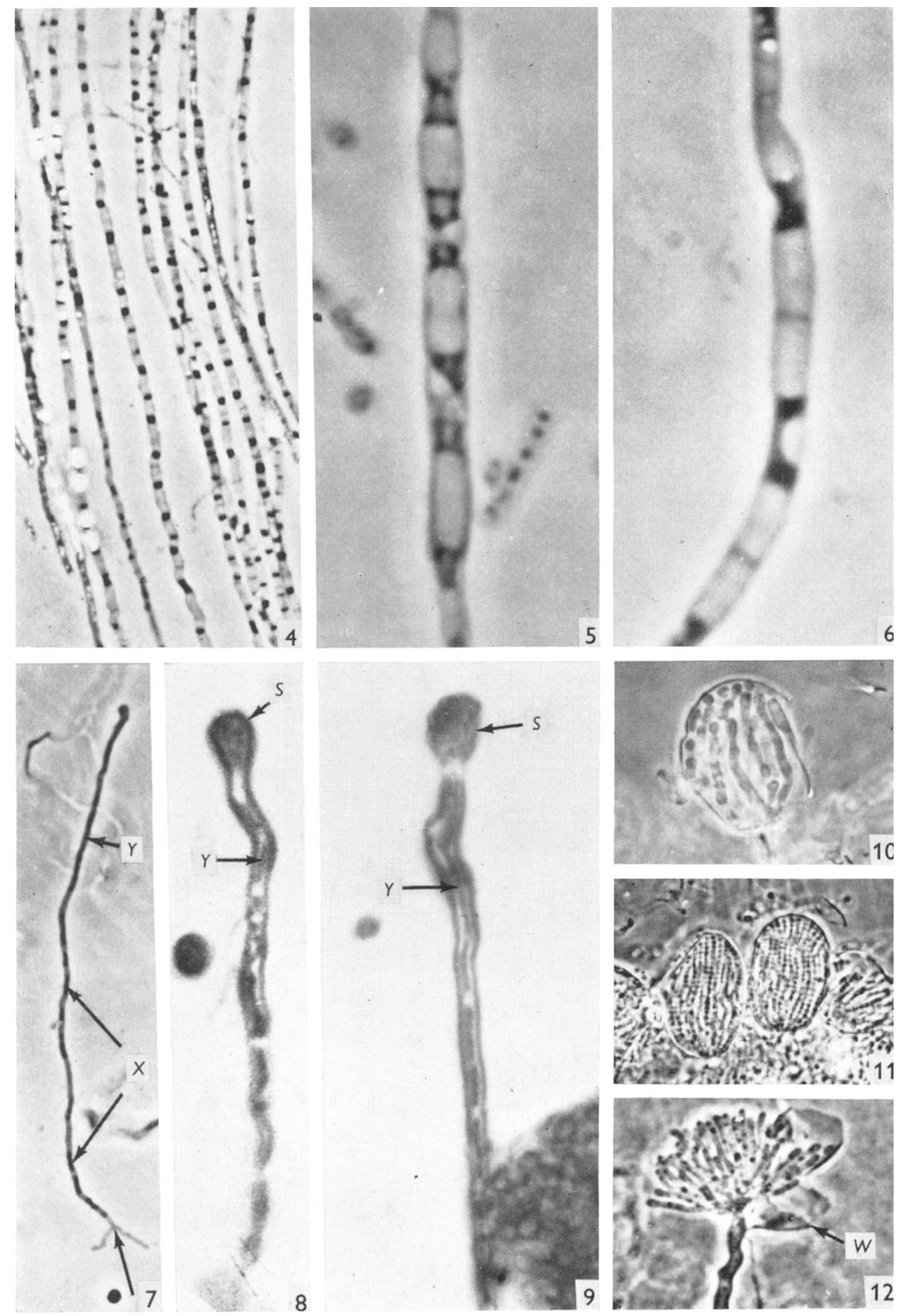

C.E. BLAND AND J, N. COUCH

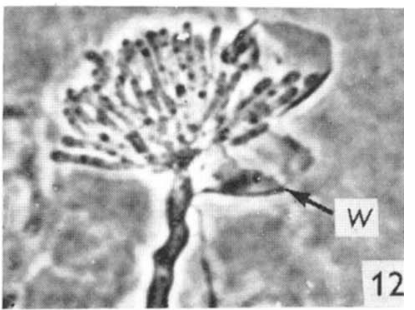


Journal of General Microbiology, Vol. 53, No. I

Plate 3
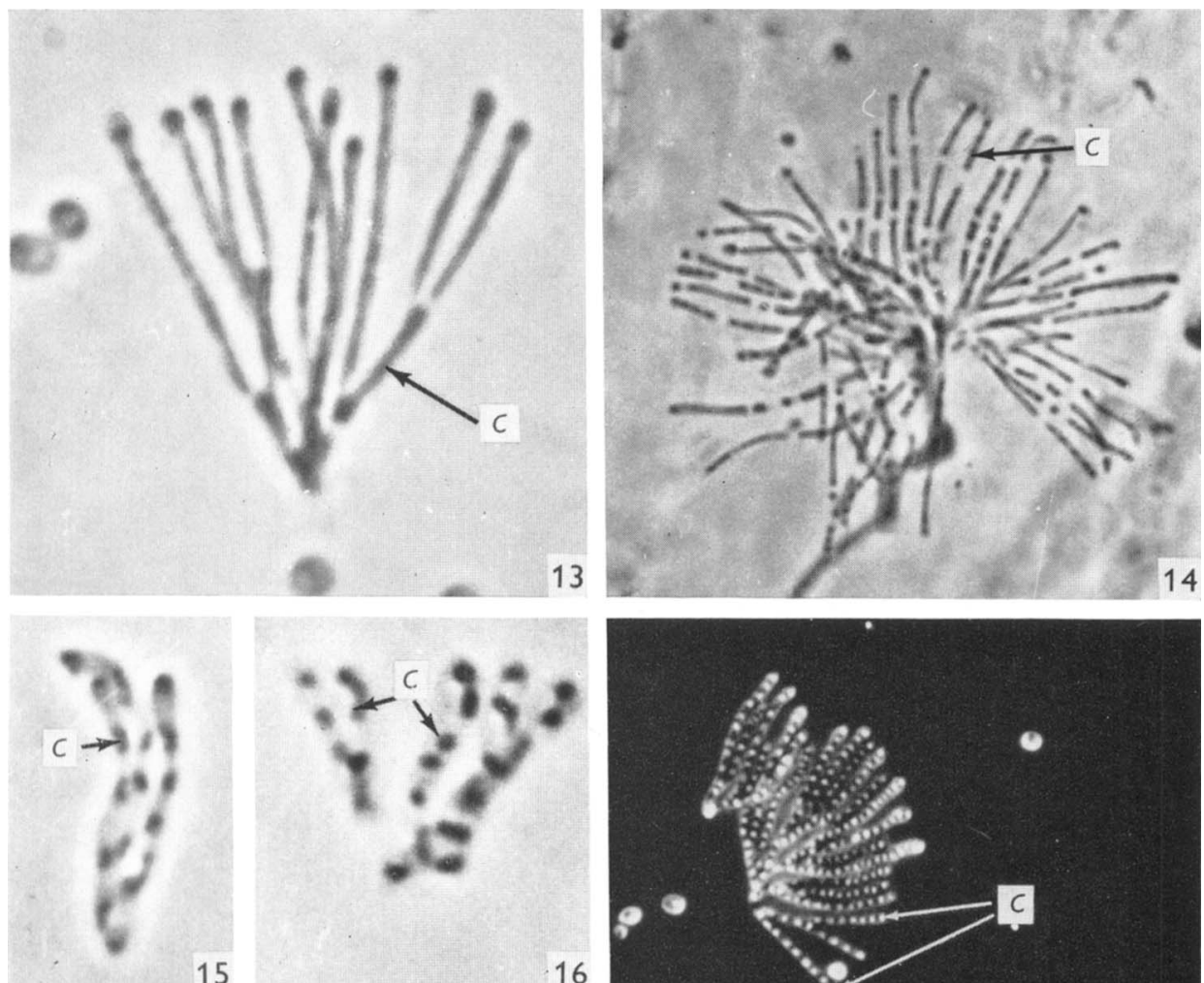

15

16
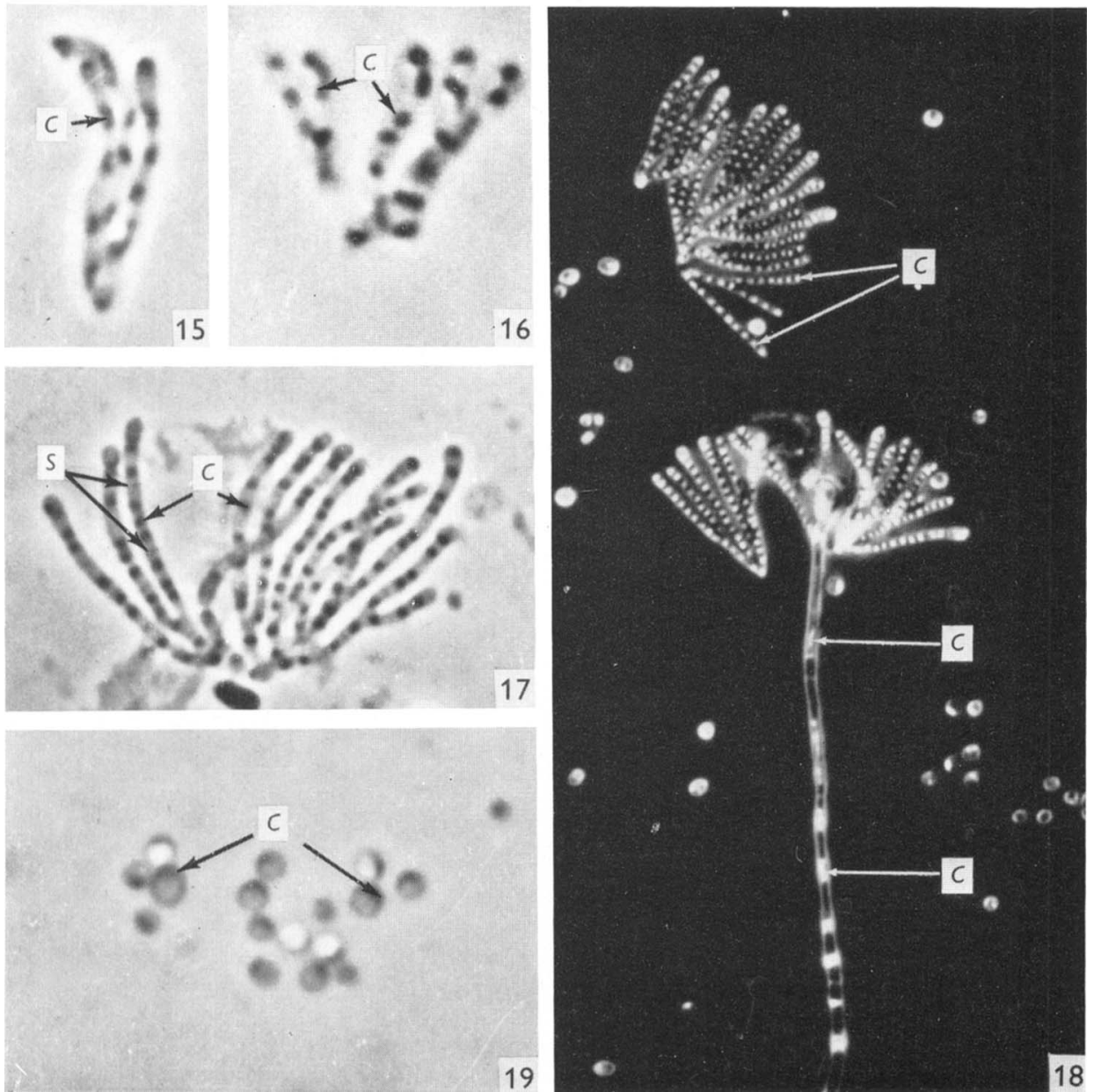

C. E. BLAND AND J. N. COUCH 

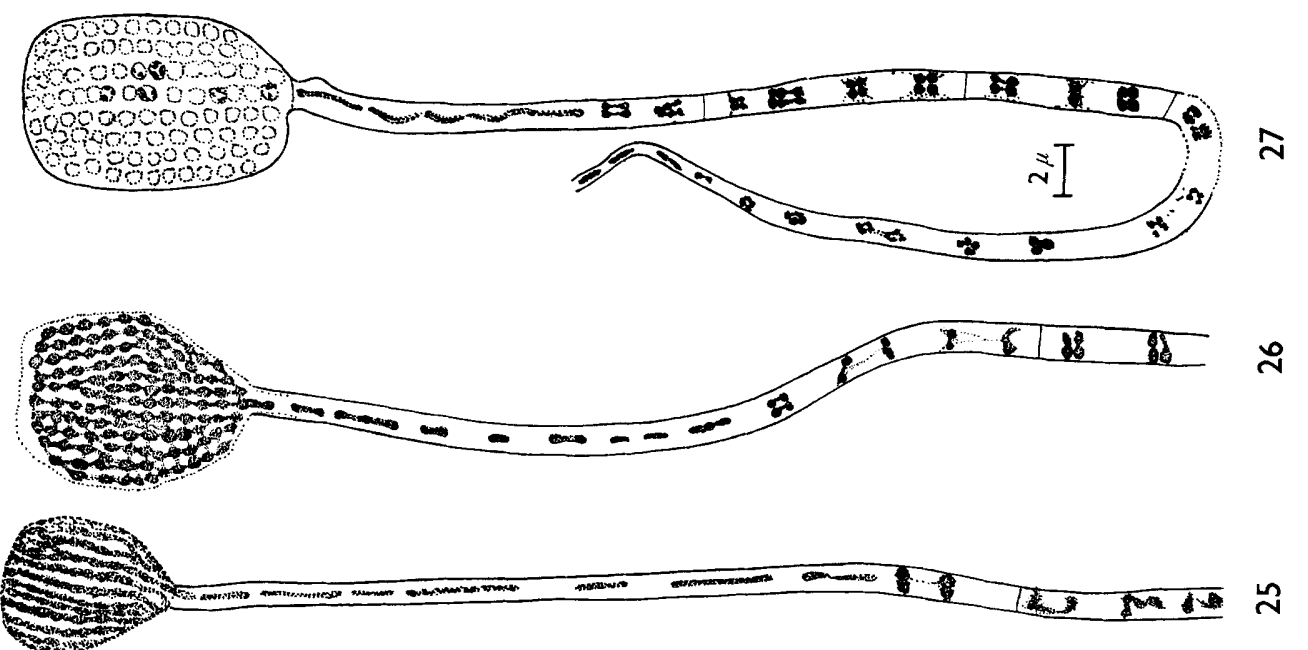

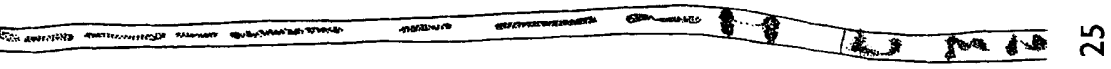
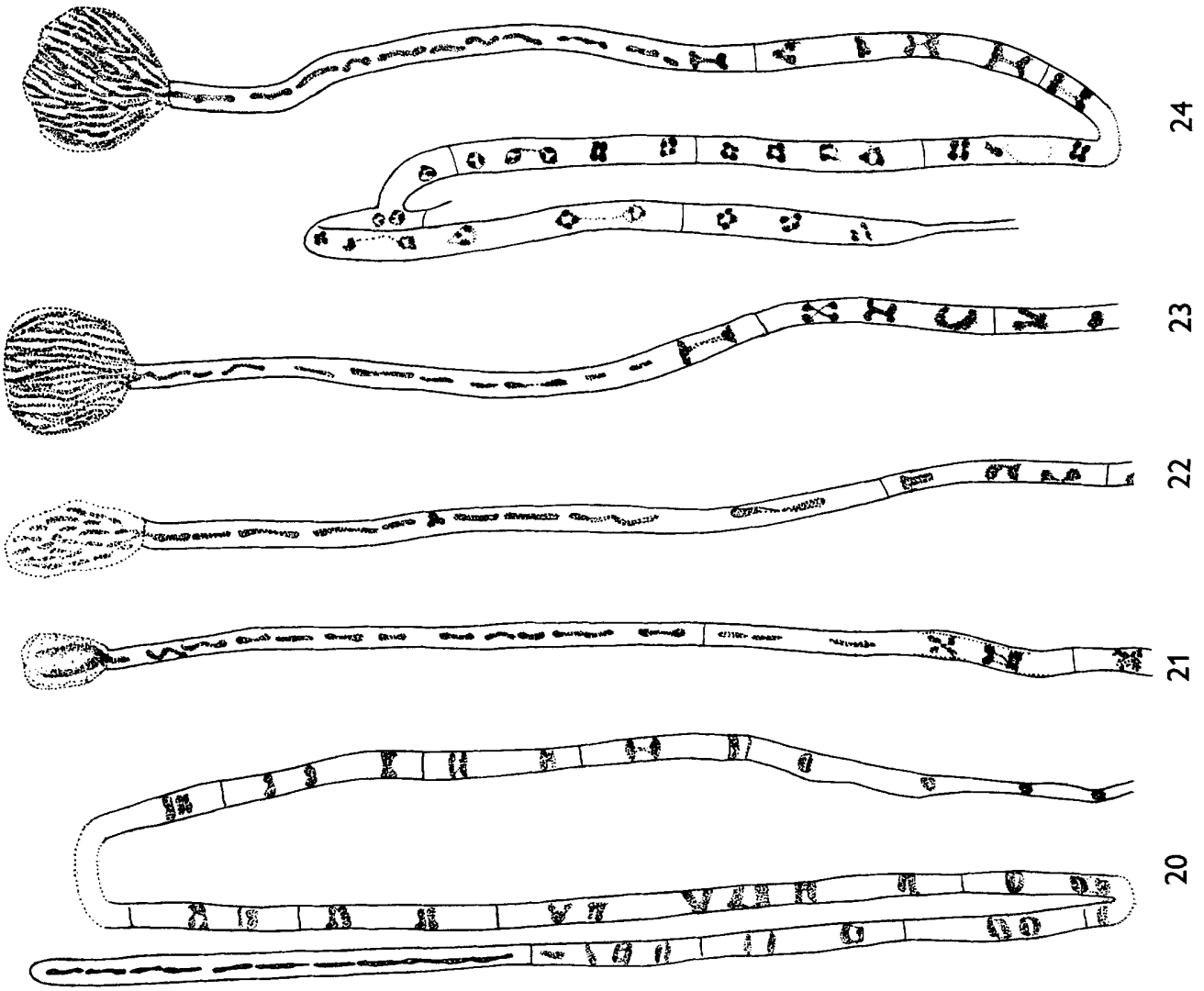

C. E. BLAND AND J. N. COUCH 